\title{
Optimization on Preparation Conditions of Salidroside Liposome and Its Immunological Activity on PCV-2 in Mice
}

\author{
Yibo Feng, ${ }^{1}$ Xiaojuan Zhao, ${ }^{2}$ Fang Lv, ${ }^{2}$ Jinqiu Zhang, ${ }^{2}$ Bihua Deng, ${ }^{2}$ Yanhong Zhao, ${ }^{2}$ \\ Yuanliang Hu, ${ }^{1}$ Deyun Wang, ${ }^{1}$ Jiaguo Liu, ${ }^{1}$ Yu Lu, ${ }^{2}$ Ruonan Bo, ${ }^{1}$ and Zhenguang Liu ${ }^{1}$ \\ ${ }^{1}$ Institute of Traditional Chinese Veterinary Medicine, College of Veterinary Medicine, Nanjing Agricultural University, \\ Nanjing 210095, China \\ ${ }^{2}$ National Research Center of Veterinary Biologicals Engineering and Technology, Jiangsu Academy of Agricultural Science, \\ Nanjing 210014, China
}

Correspondence should be addressed to Deyun Wang; dywang@njau.edu.cn and Yu Lu; luyu_nj@163.com

Received 4 February 2015; Revised 26 February 2015; Accepted 27 February 2015

Academic Editor: Michał Tomczyk

Copyright (C) 2015 Yibo Feng et al. This is an open access article distributed under the Creative Commons Attribution License, which permits unrestricted use, distribution, and reproduction in any medium, provided the original work is properly cited.

\begin{abstract}
The aim of this study was to optimize the preparation conditions of salidroside liposome with high encapsulation efficiency (EE) and to study the immunological enhancement activity of salidroside liposome as porcine circovirus type 2 virus (PCV-2) vaccine adjuvant. Response surface methodology (RSM) was selected to optimize the conditions for the preparation of salidroside liposome using Design-Expert V8.0.6 software. Three kinds of salidroside liposome adjuvants were prepared to study their adjuvant activity. $\mathrm{BALB} / \mathrm{c}$ mice were immunized with PCV-2 encapsulated in different kinds of salidroside liposome adjuvants. The PCV-2-specific IgG in immunized mice serum was determined with ELISA. The results showed that when the concentration of ammonium sulfate was $0.26 \mathrm{~mol} \cdot \mathrm{L}^{-1}$, ethanol volume $6.5 \mathrm{~mL}$, temperature $43^{\circ} \mathrm{C}$, ethanol injection rate $3 \mathrm{~mL} \cdot \mathrm{min}^{-1}$, and salidroside liposome could be prepared with high encapsulation efficiency of $94.527 \%$. Salidroside liposome as adjuvant could rapidly induce the production of PCV-2-specific IgG and salidroside liposome I adjuvant proved to provide the best effect among the three kinds of salidroside liposome adjuvants.
\end{abstract}

\section{Introduction}

Rhodiola rosea is mainly distributed in the northern hemisphere Himalayas, northwest Asia and North America, growing at an altitude of $1600-4000 \mathrm{~m}$ alpine, dry, strong ultraviolet radiation, and large temperature region, with a strong environmental adaptability and vitality [1]. Rhodiola rosea has been used as a herbal medicine for a long time and recorded in both Four Medical Classics and Compendium of Materia Medica. Pharmacological studies have shown that its main pharmacological active ingredient was salidroside. In recent years, it was reported that salidroside had antifatigue, antiaging, immune regulation, scavenging free radicals, enhancing memory, improving sleep and other pharmacological effects [2-4].

Liposomes are closed vesicles composed of a lipid bilayer and used as drug carriers in 1971. As drug carriers, liposomes had many superior characteristics in terms of improving the efficacy. Firstly, targeting liposomes were easy to locate in the kidney, liver, spleen, and other mononuclear phagocytes rich organs, and liposomes may be regarded as foreign substances in the body and could be engulfed by mononuclear phagocytes $[5,6]$. Secondly, drugs entrapped in liposomes could be released slowly, and liposomes slowed down metabolism and excretion of drugs and prolonged action of the drug [7]. In addition, liposomes were mainly engulfed by macrophages from reticuloendothelial system, which were concentrated in the liver, spleen, and bone marrow. Compared to the free drug, drug encapsulated in liposome made drug accumulation in heart and kidney significantly lower. Thus, it may decrease the toxicity when drugs were encapsulated inliposomes [8]. Lastly, the stability of the unstable drug could be improved when it was encapsulated and protected by the liposome bilayer membrane [9].

The immunologic adjuvant properties of liposomes were first studied by Allison and Gregoriadis 40 years ago [10]. 
In recent years, liposomes are often employed as delivery systems for drugs or vaccine antigens to improve cellular or tissue targeting and reduce nonspecific toxicity (drug delivery) or to minimize antigen loss [11]. Thus, it was important/necessary to do some research on new formulations of salidroside and liposome to improve immunological activity and reduce dose for clinical application.

In this study, salidroside was encapsulated with liposome by ammonium sulfate gradient method and the preparation conditions were optimized by RSM method. In the optimal condition, the porcine circovirus type 2 virus (PCV-2) was encapsulated with liposome and salidroside formulation and the immunity effect of the salidroside liposome adjuvant PCV-2 vaccine was investigated. The aim of the study is to investigate whether the immunity effect of PCV-2 can be promoted after the PCV-2 was encapsulated with salidroside liposome.

\section{Materials and Methods}

2.1. Preparation of Salidroside Liposome. The preparation of salidroside liposome was used by ammonium sulfate gradient method. Briefly, Soybean phospholipids (Shanghai Taiwei phospholipid Ltd, China), cholesterol (Tianjin Bodi Chemical Co., Ltd, China), and Tween-80 (Shanghai Dinghao Trading Co, China) were added into ethanol at a mass ratio of $6: 1: 1(\mathrm{w} / \mathrm{w} / \mathrm{w})$ and dissolved with appropriate ultrasound to get a stable solution. The solution was poured into ammonium sulfate solution at a constant speed. Then, the ethanol was removed by vacuum rotary evaporator. Ammonium sulfate in external aqueous phase was removed after 24 hours of dialysis in PBS ( $\mathrm{pH}=7.4)$. A certain concentration of salidroside (Aladdin Co., Ltd) solution was mixed with blank liposome at a certain temperature and speed for $30 \mathrm{~min}$ and salidroside liposome was obtained. Finally, the salidroside liposome was filtered using $0.45 \mu \mathrm{m}$ and $0.22 \mu \mathrm{m}$ millipore membrane successively.

2.2. The Determination of Encapsulation Efficiency. Encapsulation efficiency (EE) of salidroside liposome was measured by dialysis method [12-14]. In brief, $2 \mathrm{~mL}$ of salidroside liposome was added into a dialysis bag with a hydrophilic cellulose membrane (3,000 MWCO) to remove free salidroside in PBS solution $(200 \mathrm{~mL}, \mathrm{pH}=7.4)$. After 24 hours of dialysis, $1 \mathrm{~mL}$ of salidroside liposome was placed into a $10 \mathrm{~mL}$ volumetric flask for analysis. $3 \mathrm{~mL}$ of $10 \%$ Triton X100 (Shanghai Yuanye Biotechnology Co., Ltd, China) was used to rupture salidroside liposome. $1.5 \mathrm{~mL}$ of $2 \%$ sodium carbonate solution and $1 \mathrm{~mL}$ of azide reagent were added into the liposome solution and the solution was standing for $5 \mathrm{~min}$ after shaking. $5 \%$ sodium hydroxide solution was added into the volumetric flask to $10 \mathrm{~mL}$. The amount of salidroside was measured by the chemical colorimetric technique utilizing reaction of diazotization in basic solution [15]. The formula to calculate the EE of salidroside liposome was shown below. " $C_{e}$ ” was the amount of encapsulated salidroside and " $C_{t}$ ” was the total amount of salidroside. Consider

$$
\mathrm{EE} \%=\frac{C_{\mathrm{e}} \times 100 \%}{C_{\mathrm{t}}} .
$$

TABLE 1: Levels and code of variables chosen for Box-Behnken design.

\begin{tabular}{lccc}
\hline \multirow{2}{*}{ Factors } & \multicolumn{4}{c}{ Levels and range } \\
& -1 & 0 & 1 \\
\hline$X_{1}$ : ammonium sulfate concentration $(\mathrm{mol} / \mathrm{L})$ & 0.20 & 0.25 & 0.30 \\
$X_{2}$ : ethanol injection volume $(\mathrm{mL})$ & 4 & 6 & 8 \\
$X_{3}$ : temperature $\left({ }^{\circ} \mathrm{C}\right)$ & 40 & 45 & 50 \\
$X_{4}$ : ethanol injection rate $(\mathrm{mL} / \mathrm{min})$ & 2 & 3 & 4 \\
\hline
\end{tabular}

2.3. Optimization of Salidroside Liposome Preparation Condition. According to the previous research, four factors (ammonium sulfate concentration, ethanol injection volume, temperature, and ethanol injection rate) were the main single factors affecting the EE of salidroside liposome. On the basis of single-factor test results, four main factors were determined as follows: ammonium sulfate concentration $(\mathrm{mol} / \mathrm{L})\left(X_{1}\right)$, ethanol injection volume $(\mathrm{mL})\left(X_{2}\right)$, temperature $\left({ }^{\circ} \mathrm{C}\right)\left(X_{3}\right)$, and ethanol injection rate $(\mathrm{mL} / \mathrm{min})$ $\left(X_{4}\right)$. Response surface methodology (RSM) was used to optimize the preparation conditions of salidroside liposome using Design-Expert V8.0.6 software. EE was chosen as the response $(Y), 29$ test points of a four factor-three coded level Box-Behnken design (BBD) were designed. The factors and levels were shown in Table 1.

2.4. Preparation of Salidroside Liposome Adjuvant PCV-2 Vaccines. Different concentrations of salidroside liposome were prepared according to the method mentioned in Section 2.1 with some modifications. Briefly, soybean phospholipids (300 mg, $600 \mathrm{mg}$, and $900 \mathrm{mg}$ ), cholesterol (50 mg, $100 \mathrm{mg}$, and $150 \mathrm{mg}$ ), and Tween-80 (50 mg, $100 \mathrm{mg}$, and $150 \mathrm{mg}$ ) were added into $7.0 \mathrm{~mL}$ of ethanol at a mass ratio of $6: 1: 1(\mathrm{w} / \mathrm{w} / \mathrm{w})$, and the other preparation conditions remained unchanged. Three kinds of salidroside liposome were prepared and named to salidroside liposome I, salidroside liposome II, and salidroside liposome III, respectively.

Salidroside liposomes PCV-2 were prepared by repeating freeze-thaw method [16]. In brief, salidroside liposomes were mixed with PCV-2 antigen (inactivated, virus titer: $1 \times 10^{7.5} \mathrm{TCID}_{50} / \mathrm{mL}$ ) at volume ratio of $2: 1,3: 1,4: 1,5: 1$, and $6: 1$. Each mixture was frozen for $3 \mathrm{~h}$ in $-20^{\circ} \mathrm{C}$. Then, they were transferred to a constant temperature oscillator for $30 \mathrm{~min}$ under the conditions of $45^{\circ} \mathrm{C}$ and $80 \mathrm{rpm}$. This operation was repeated 3 times.

2.4.1. Determination of Encapsulation Efficiency of PCV-2. EE of PCV-2 was determined by BCA Protein Assay Kit (Pierce Chemical Co., USA) with some modifications [12, 13, 17]. Briefly, salidroside liposome PCV-2 was mixed with Triton X100 at a temperature of $65^{\circ} \mathrm{C}$ for $10 \mathrm{~min}$ to release the antigen inside the liposome and was then centrifuged at $8500 \mathrm{~g}$ for $30 \mathrm{~min}$. The protein concentration in the supernatant was measured and denoted by $C_{t}$. Salidroside liposome PCV-2 without Triton X-100 was centrifuged at $8500 \mathrm{~g}$ for $30 \mathrm{~min}$. The protein concentration in the supernatant was measured 
by the kit and denoted by $C_{\mathrm{f}}$. The EE of antigen was calculated according to the following formula:

$$
\mathrm{EE}=\frac{\left(C_{\mathrm{t}}-C_{\mathrm{f}}\right) \times 100 \%}{C_{\mathrm{t}}} .
$$

2.4.2. Characteristics of Salidroside Liposome PCV-2. The particle sizes of salidroside liposome PCV-2 were measured by Hydro $2000 \mathrm{Mu}$ laser particle size analyzer (Hydro2000Mu, MAL 1009117, Malvern Instruments Ltd.). The morphology of salidroside liposome PCV-2 was observed by transmission electron microscope (Tecnai 12, Holland).

2.4.3. In Vitro Release Assay. In order to determine the release of PCV-2 from salidroside liposome PCV-2, $200 \mu \mathrm{L}$ of the salidroside liposome PCV-2 was mixed with $800 \mu \mathrm{L}$ of PBS ( $\mathrm{pH} 7.4$ ) and maintained at $37^{\circ} \mathrm{C}$ with a circulating water bath and constantly stirred by a magnetic stirrer at $150 \mathrm{rpm}$. At different time intervals, the PCV-2 concentration in PBS solution was determined using BCA Protein Assay Kit (Pierce Chemical Co., USA). The release experiments were performed in triplicate.

\subsection{Immunological Activity of Salidroside Liposome PCV-2 in Mice}

2.5.1. Animals. Four-week-old BALB/c mice were purchased from Comparative Medicine Centre of Yangzhou University and acclimatized for 7 days prior to immunization. The mice were maintained under controlled conditions at temperature of $24 \pm 1^{\circ} \mathrm{C}$, humidity of $50 \pm 10 \%$, and a $12 / 12$-h light-dark cycle with free access to food and water. Each mouse was used once and treated in accordance with the National Institutes of Health guide lines for the care and use of laboratory animals.

2.5.2. Mouse Immunization. Mice were injected subcutaneously in the dorsal skinfold on day 0 with $0.2 \mathrm{~mL}$ different salidroside liposome adjuvants PCV-2 vaccines, Montanide ISA 201 adjuvant PCV-2 vaccines and PCV-2 vaccines. A booster dose was given to each primed mouse 14 days after the first immunization. Blood samples of all groups (6 mice per group) were collected for antibody titer assays on 1, 3, 5, $7,9,11$, and 13 weeks after the second immunization.

2.5.3. Serum PCV-2-Specific IgG Assay. PCV-2-specific IgG in the serum was determined with enzyme linked immunosorbent assay (ELISA). ELISA kit to detect the antibody against PCV-2 (Wuhankeqian Animal Biological Products Co., Ltd, China) was selected for the serum PCV-2-specific IgG assay, and all operations were carried out in accordance with the kit instructions.

The serum isolated from all groups in a 1:1600 dilution was used for PCV-2-specific IgG determination, and 1:5000 goat anti-mouse total IgG-HRP conjugate (Jackson Immuno Research, USA) was used as the second antibody. The absorbance was measured at $630 \mathrm{~nm}$ using a STAT FAX 2100 microplate reader (AWARENESS, USA).
2.6. Statistical Analysis. Data of the optimization of salidroside liposome preparation were analyzed using the DesignExpert program and second-order polynomial equation, and ANOVA of the quadratic regression model, and optimal conditions were shown. Results in this study were presented as mean \pm standard errors (S.E.). Statistical comparisons were made by the LSD and Duncan's multiple range test in SPSS (version 19.0), and differences between groups were considered significant if the $P$ value was less than 0.05 .

\section{Results}

3.1. Statistical Analysis and Model Fitting. There were a total of 29 runs for optimizing the four individual parameters in the $\mathrm{BBD}$, and the experimental conditions and the EE of salidroside liposome according to the factorial design were shown in Table 2, which also included the predicted values.

The quadratic model was found to be the fitting model for the EE of salidroside liposome. An empirical relationship expressed by a second-order polynomial equation with interaction terms was fitted between obtained experimental results. The EE obtained in terms of coded factors is calculated as follows:

$$
\begin{aligned}
Y= & -221.1667+46.6667 X_{1}+19.4000 X_{2} \\
& +11.5217 X_{3}+0.0917 X_{4}+13.7500 X_{1} X_{2} \\
& +3.4000 X_{1} X_{3}-0.0550 X_{2} X_{3}-0.3750 X_{2} X_{4} \\
& +0.8200 X_{3} X_{4}-540.0000 X_{1}{ }^{2}-1.5188 X_{2}{ }^{2} \\
& -0.1675 X_{3}{ }^{2}-5.7125 X_{4}{ }^{2} .
\end{aligned}
$$

It was shown that the test chosen quadratic model was highly significant $\left(P_{\text {model }}<0.0001\right)$ through the analysis of variance of the model from Table 3, and lack of fit was not significant $(P=0.2735>0.05)$. Except $X_{1} X_{4}$ (ammonium sulfate concentration and ethanol injection speed interaction) in the model, the " $P$ " values of all quadratics were less than 0.05 , indicating that all the models except $X_{1} X_{4}$ have significant effects on liposome encapsulation efficiency. According to correlation $R^{2}=0.9954$ and the correction coefficient of determination $R_{\mathrm{Adj}}{ }^{2}=0.9906$, only about $1 \%$ of the total variation of liposome encapsulation efficiency could not be explained. In summary, the result showed good fit of the model.

Results of the EE of salidroside liposome affected by ammonium sulfate concentration $(\mathrm{mol} / \mathrm{L})$, ethanol injection volume $(\mathrm{mL})$, temperature $\left({ }^{\circ} \mathrm{C}\right)$, and ethanol injection rate $(\mathrm{mL} / \mathrm{min})$ were presented in Figure 1. These types of plots showed the effects of two factors on the response at a time and the other factors were maintained at the zero level.

To show the relationship between responses and experiment levels of each variable, the response surface (3D) and contour plots are displayed in Figure 1, respectively. The shapes of the contour plots indicate whether the mutual interactions between the variables are significant or not. Circular contour plot indicates that the interactions between the corresponding variables are negligible, while elliptical 
TABLE 2: The design and results of Box-Behnken experiment.

\begin{tabular}{|c|c|c|c|c|c|c|}
\hline \multirow{2}{*}{ Number } & \multicolumn{4}{|c|}{ Levels of independent factors } & \multicolumn{2}{|c|}{ Response: EE (\%) } \\
\hline & $X_{1}$ & $X_{2}$ & $X_{3}$ & $X_{4}$ & Predicted acquired EE & Practical acquired EE \\
\hline 1 & 0.30 & 6 & 45 & 4 & 88.50 & 88.36 \\
\hline 2 & 0.30 & 4 & 45 & 3 & 84.10 & 84.38 \\
\hline 3 & 0.25 & 6 & 40 & 2 & 91.20 & 91.18 \\
\hline 4 & 0.25 & 6 & 45 & 3 & 94.70 & 84.55 \\
\hline 5 & 0.20 & 4 & 45 & 3 & 85.70 & 85.92 \\
\hline 6 & 0.25 & 6 & 50 & 2 & 76.80 & 77.27 \\
\hline 7 & 0.25 & 8 & 45 & 4 & 84.90 & 94.60 \\
\hline 8 & 0.20 & 8 & 45 & 3 & 87.30 & 87.65 \\
\hline 9 & 0.25 & 6 & 45 & 3 & 94.10 & 94.60 \\
\hline 10 & 0.30 & 6 & 50 & 3 & 87.90 & 87.65 \\
\hline 11 & 0.30 & 6 & 40 & 3 & 91.30 & 91.69 \\
\hline 12 & 0.25 & 4 & 40 & 3 & 85.10 & 84.63 \\
\hline 13 & 0.30 & 6 & 45 & 2 & 88.20 & 87.93 \\
\hline 14 & 0.20 & 6 & 40 & 3 & 92.20 & 92.17 \\
\hline 15 & 0.20 & 6 & 45 & 2 & 86.00 & 86.21 \\
\hline 16 & 0.25 & 6 & 45 & 3 & 94.60 & 94.60 \\
\hline 17 & 0.20 & 6 & 50 & 3 & 85.40 & 84.47 \\
\hline 18 & 0.25 & 8 & 45 & 2 & 85.60 & 85.12 \\
\hline 19 & 0.25 & 4 & 45 & 2 & 79.50 & 79.57 \\
\hline 20 & 0.25 & 8 & 40 & 3 & 89.40 & 89.78 \\
\hline 21 & 0.25 & 8 & 50 & 3 & 82.40 & 82.95 \\
\hline 22 & 0.30 & 8 & 45 & 3 & 91.20 & 91.20 \\
\hline 23 & 0.25 & 4 & 45 & 4 & 81.80 & 82.00 \\
\hline 24 & 0.20 & 6 & 45 & 4 & 87.30 & 87.22 \\
\hline 25 & 0.25 & 4 & 50 & 3 & 80.30 & 80.00 \\
\hline 26 & 0.25 & 6 & 40 & 4 & 84.20 & 83.93 \\
\hline 27 & 0.25 & 6 & 50 & 4 & 86.20 & 86.40 \\
\hline 28 & 0.25 & 6 & 45 & 3 & 94.50 & 94.60 \\
\hline 29 & 0.25 & 6 & 45 & 3 & 95.10 & 94.60 \\
\hline
\end{tabular}

contour plot indicates that the interactions between the corresponding variables are significant [18]. The maximum value predicted by the surface was confined in the smallest ellipse in the contour diagram. The elliptical contours were obtained when there was a perfect interaction between the independent variables [19].

The response surface (3D) and contour plot as in Figure 1(a) made EE as a function of ammonium sulfate concentration and ethanol injection volume at fixed temperature and ethanol injection rate. It is indicated that EE rapidly increased with an increase in ethanol injection volume from 4 to $7 \mathrm{~mL}$. In addition, EE increased with an increase in ammonium sulfate concentration from 0.20 to $0.28 \mathrm{~mol} / \mathrm{L}$ and then reached a plateau region.

Figure 1(b) depicted response surface (3D) and contour plot of the effects of the two variables, namely, the interaction effect of ammonium sulfate concentration and temperature on the EE of salidroside liposome. It indicates that EE gradually increased with an increase in temperature from 40 to $44^{\circ} \mathrm{C}$. In addition, EE increased with an increase in ammonium sulfate concentration from 0.20 to $0.28 \mathrm{~mol} / \mathrm{L}$ and then reached a plateau region. However, the EE was slightly changed after $0.28 \mathrm{~mol} / \mathrm{L}$.

The response surface plot (3D) and the contour plot at varying ammonium sulfate concentration and ethanol injection rate at fixed temperature and ethanol injection volume are depicted. As shown in Figure 1(c), the EE was gradually increased before the ethanol injection rate was $3 \mathrm{~mL} / \mathrm{min}$. The same variation was found when it came to ammonium sulfate concentration.

Interaction of ethanol volume and temperature was displayed in the $3 \mathrm{D}$ response surface plot and the contour plot. According to the results in Figure 1(d), EE gradually increased with an increase in ethanol volume from 4 to $7 \mathrm{~mL}$. In addition, $\mathrm{EE}$ increased with an increase in temperature from 40 to $45^{\circ} \mathrm{C}$ and then reached a plateau region. However, the EE was slightly decreased after $44^{\circ} \mathrm{C}$.

Figure 1(e) represented the effects of ethanol volume and ethanol injection rate on the $\mathrm{EE}$ of salidroside liposome, while ammonium sulfate concentration and temperature were fixed. Both ethanol volume and ethanol injection rate had significant effects on EE since the contour plot was 
TABLE 3: ANOVA for response surface quadratic model.

\begin{tabular}{|c|c|c|c|c|c|c|}
\hline Source & Sum of squares & $\mathrm{df}$ & Mean Square & $F$ value & $P$ value Prob $>F$ & Significance \\
\hline Model & 657.64 & 14 & 657.64 & 216.15 & $<0.0001$ & $\operatorname{Sig}^{* * *}$ \\
\hline$X_{1}$ & 4.44 & 1 & 4.44 & 20.43 & 0.0005 & $* *$ \\
\hline$X_{2}$ & 49.21 & 1 & 49.21 & 226.43 & $<0.0001$ & $* * *$ \\
\hline$X_{3}$ & 98.61 & 1 & 98.61 & 453.27 & $<0.0001$ & $* * *$ \\
\hline$X_{4}$ & 2.61 & 1 & 2.61 & 12.03 & 0.0038 & $* *$ \\
\hline$X_{1} X_{2}$ & 7.56 & 1 & 7.56 & 34.80 & $<0.0001$ & $* * *$ \\
\hline$X_{1} X_{3}$ & 2.89 & 1 & 2.89 & 13.30 & 0.0026 & $* *$ \\
\hline$X_{1} X_{4}$ & 0.25 & 1 & 0.25 & 1.15 & 0.3016 & \\
\hline$X_{2} X_{3}$ & 1.21 & 1 & 1.21 & 5.57 & 0.0333 & * \\
\hline$X_{2} X_{4}$ & 2.25 & 1 & 2.25 & 10.35 & 0.0062 & $* *$ \\
\hline$X_{3} X_{4}$ & 67.24 & 1 & 67.24 & 309.40 & $<0.0001$ & $* * *$ \\
\hline$X_{1}^{2}$ & 11.82 & 1 & 11.82 & 54.40 & $<0.0001$ & $* * *$ \\
\hline$X_{2}{ }^{2}$ & 239.39 & 1 & 239.39 & 1101.54 & $<0.0001$ & $* * *$ \\
\hline$X_{3}{ }^{2}$ & 113.74 & 1 & 113.74 & 523.38 & $<0.0001$ & $* * *$ \\
\hline$X_{4}{ }^{2}$ & 211.67 & 1 & 211.67 & 974.00 & $<0.0001$ & $* * *$ \\
\hline Residual & 3.04 & 14 & 0.22 & & & \\
\hline Lack of fit & 2.52 & 10 & 0.25 & 1.94 & 0.2735 & Not sig. \\
\hline Pure error & 0.52 & 4 & 0.13 & & & \\
\hline Cor. total & 660.68 & 28 & & & & \\
\hline \multicolumn{7}{|c|}{$R^{2}=0.9954, R_{\mathrm{Adj}}^{2}=0.9906$, and $R_{\text {Pred }}{ }^{2}=0.9768$} \\
\hline
\end{tabular}

${ }^{*} P<0.05 ;{ }^{* *} P<0.01 ;{ }^{* * *} P<0.001$.

elliptical. The maximum EE could be achieved when the ethanol injection rate and ethanol volume were within the level range from 3 to $3.5 \mathrm{~mL} / \mathrm{min}$ and nearly $7 \mathrm{~mL}$.

The interaction effects of temperature and ethanol injection rate were shown in Figure 1(f). The EE gradually increased with an increase in temperature from 40 to $44^{\circ} \mathrm{C}$. In addition, EE increased with an increase in ethanol injection rate from 2 to $3 \mathrm{~mL} / \mathrm{min}$ and then reached a plateau region where the EE was slightly decreased after $3 \mathrm{~mL} / \mathrm{min}$.

3.2. Verification of the Predictive Mode. To further verify the accuracy of predictions, the model equation for predicting the optimum response values was rechecked under the selected optimal conditions. Additional experiments for reconfirmation were performed under the selected optimal conditions: ammonium sulfate concentration of $0.26 \mathrm{~mol} / \mathrm{L}$, ethanol injection volume of $6.5 \mathrm{~mL}$, a temperature of $43^{\circ} \mathrm{C}$, and ethanol injection rate of $3 \mathrm{~mL} / \mathrm{min}$.

The optimum conditions above were considered to be optimum according to the RSM. In our study, a set of optimum conditions were validated experimentally and the values of the responses using the model equation were predicted. A mean EE value of $94.527 \pm 0.682 \%(n=3)$ was obtained from practical experiments. The mean EE value was close to the predicted value of $95.422 \%$, which demonstrated the validation of the RSM model.

3.3. Results of Encapsulation Efficiency of PCV-2. The results measured by BCA Protein Assay Kit were shown in Table 4. The data showed that when salidroside liposome I PCV2, salidroside liposome II PCV-2, and salidroside liposome III PCV-2 had the same amount of PCV-2 antigen,
$1.1 \times 10^{7} \mathrm{TCID}_{50} / 0.2 \mathrm{~mL}$, and all the salidroside liposome PCV-2 could get the maximum encapsulation efficiency of PCV-2 when the volume ratio of salidroside liposome to PCV-2 was $5: 1$. Thus, $5: 1$ volume ratio of salidroside liposome to PCV-2 was selected for the preparation of salidroside liposome PCV-2.

3.4. Results of Characteristics of Salidroside Liposome Adjuvant PCV-2 Vaccines. The particle sizes of three different liposomes were, respectively, $138 \pm 22 \mathrm{~nm}$ (Salidroside liposome I), $165 \pm 18 \mathrm{~nm}$ (Salidroside liposome II), and $157 \pm$ $12 \mathrm{~nm}$ (Salidroside liposome III) (Table 5). The transmission electron microscope of salidroside liposome PCV-2 was shown in Figure 2. The salidroside liposome PCV-2 presented the homogeneous milk-white and translucent suspension. By transmission electron micrograph, salidroside liposome PCV-2 appeared nearly spherical shape and the monodisperse vesicles. Taken these results together, it was clear that liposomes were of the small particle size and uniform distribution.

3.5. In Vitro Release of PCV-2 from Salidroside Liposome PCV2. In vitro, the release of three kinds of salidroside liposome PCV-2 from salidroside liposome was shown in Figure 3. In the early period, the release of salidroside liposome II PCV-2 was the slowest. After 4 days, the release of salidroside liposome II PCV-2 was faster than that of salidroside liposome I PCV-2 and slower than that of salidroside liposome III PCV2.

3.6. Salidroside Liposome as Adjuvant Could Promote the Production of PCV-2-Specific IgG. The serum PCV-2-specific 


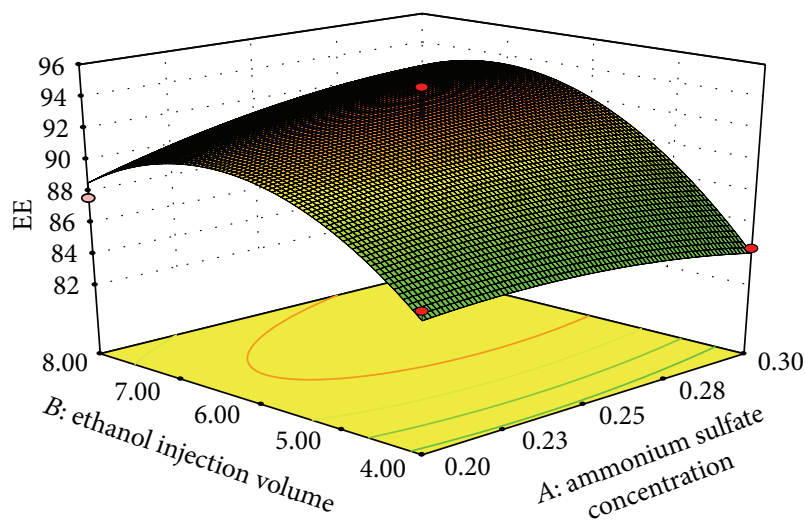

Design-Expert V8.0.6 software

Factor coding: actual

EE

- Design points above predicted value

- Design points below predicted value

$\|_{77}^{95}$

$X_{1}=A$ : ammonium sulfate concentration

$X_{2}=B$ : ethanol injection volume

Actual factors

$C$ : temperature $=45.00$

$D$ : ethanol injection rate $=3.00$

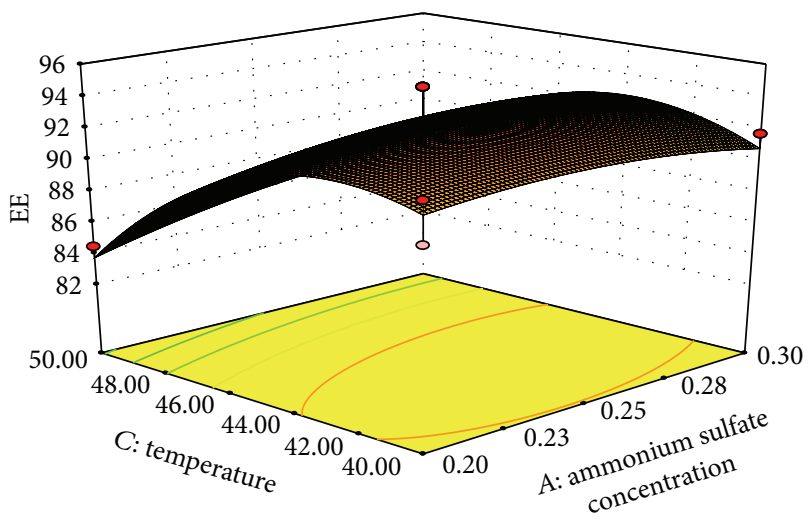

Design-Expert V8.0.6 software

Factor coding: actual

$\mathrm{EE}$

- Design points above predicted value

- Design points below predicted value

$\prod_{77}^{95}$

$X_{1}=A$ : ammonium sulfate concentration

$X_{2}=$ C: temperature

Actual factors

$B:$ ethanol injection volume $=6.00$

$D$ : ethanol injection rate $=3.00$

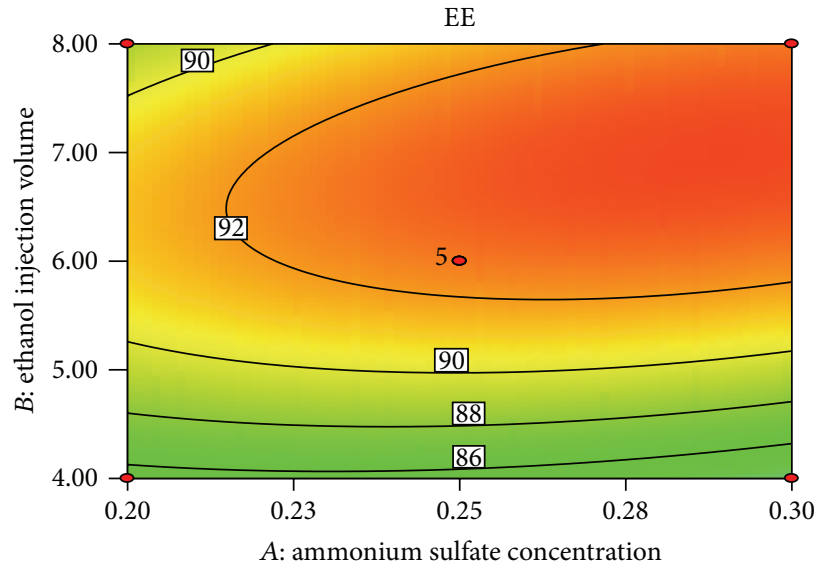

Design-Expert V8.0.6 software

Factor coding: actual

$\mathrm{EE}$

- Design points

$\prod_{77}^{95}$

$X_{1}=A$ : ammonium sulfate concentration

$X_{2}=B$ : ethanol injection volume

Actual factors

$C$ : temperature $=45.00$

$D$ : ethanol injection rate $=3.00$

(a)

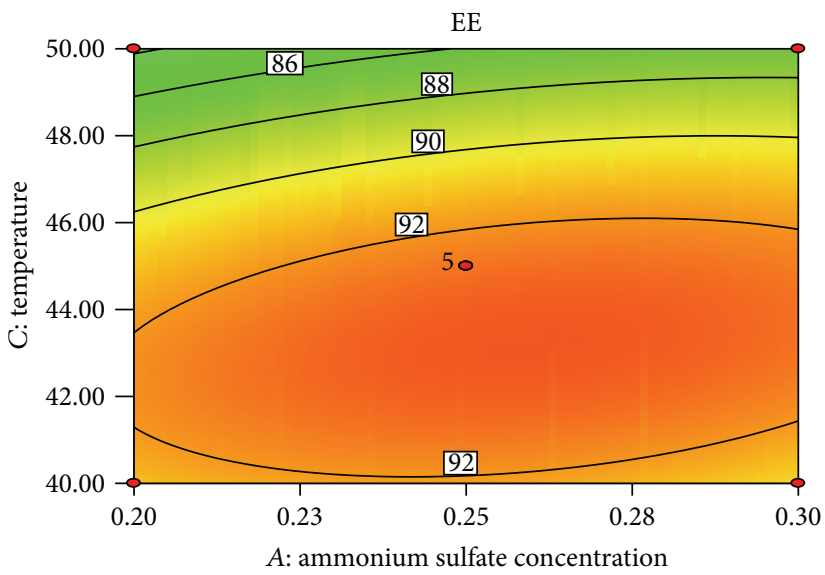

Design-Expert V8.0.6 software

Factor coding: actual

$\mathrm{EE}$

- Design points

$\prod_{77}^{95}$

$X_{1}=A$ : ammonium sulfate concentration

$X_{2}=$ C: temperature

Actual factors

$B:$ ethanol injection volume $=6.00$

$D$ : ethanol injection rate $=3.00$

(b) 


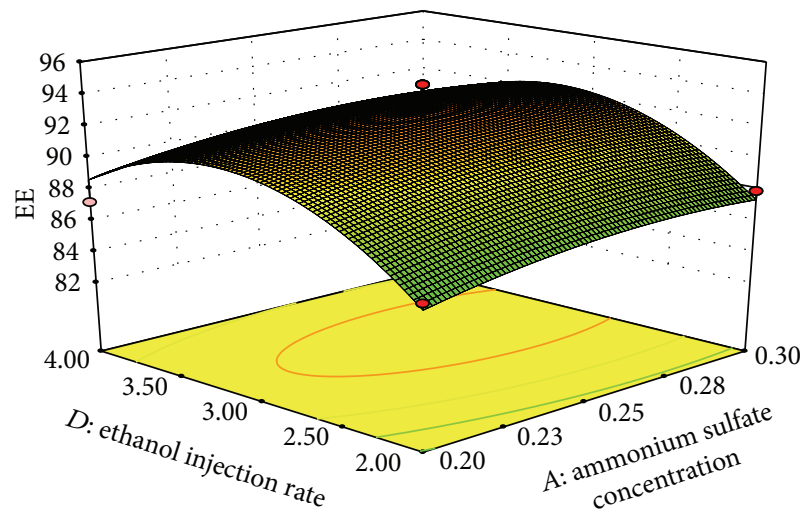

Design-Expert V8.0.6 software

Factor coding: actual

EE

- Design points above predicted value

- Design points below predicted value

$\|_{77}^{95}$

$X_{1}=A$ : ammonium sulfate concentration

$X_{2}=D$ : ethanol injection rate

Actual factors

$B:$ ethanol injection volume $=6.00$

C: temperature $=45.00$

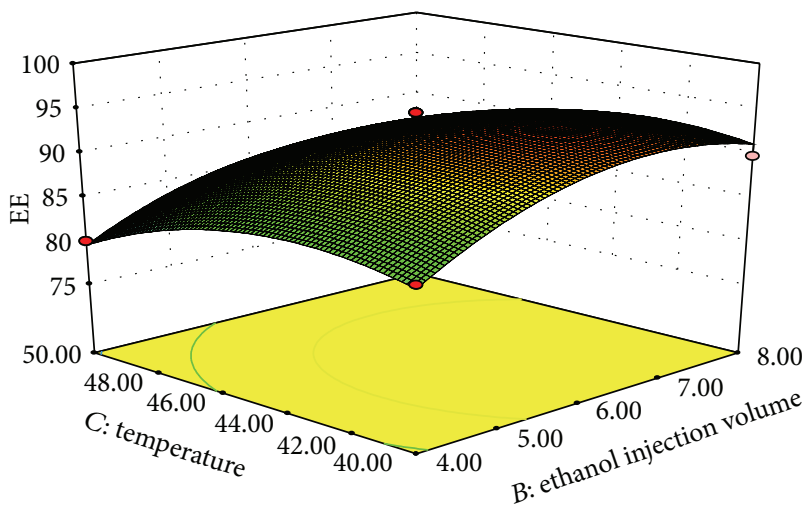

Design-Expert V8.0.6 software

Factor coding: actual

$\mathrm{EE}$

- Design points above predicted value

- Design points below predicted value

$\prod_{77}^{95}$

$X_{1}=B$ : ethanol injection volume

$X_{2}=C:$ temperature

Actual factors

A: ammonium sulfate concentration $=0.25$

$D$ : ethanol injection rate $=3.00$

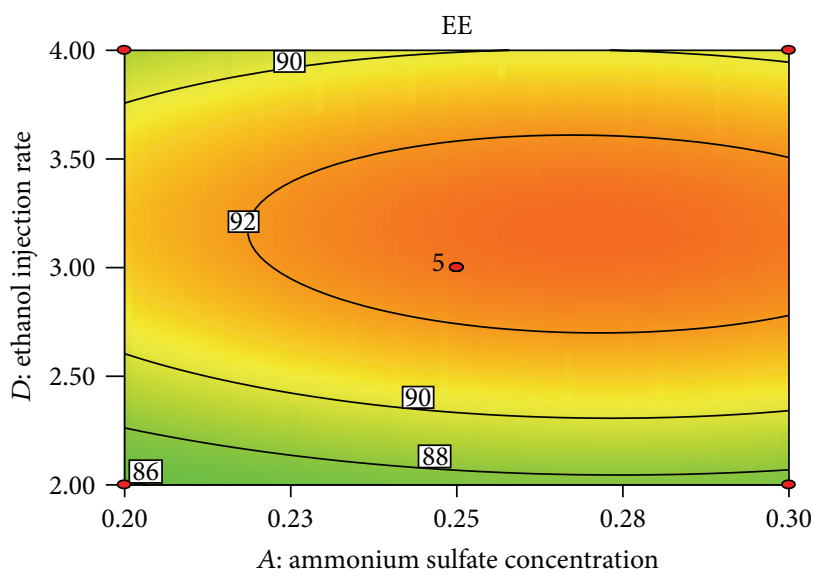

Design-Expert V8.0.6 software

Factor coding: actual

EE

- Design points

95
77

$X_{1}=A$ : ammonium sulfate concentration

$X_{2}=D$ : ethanol injection rate

Actual factors

$B$ : ethanol injection volume $=6.00$

$C$ : temperature $=45.00$

(c)

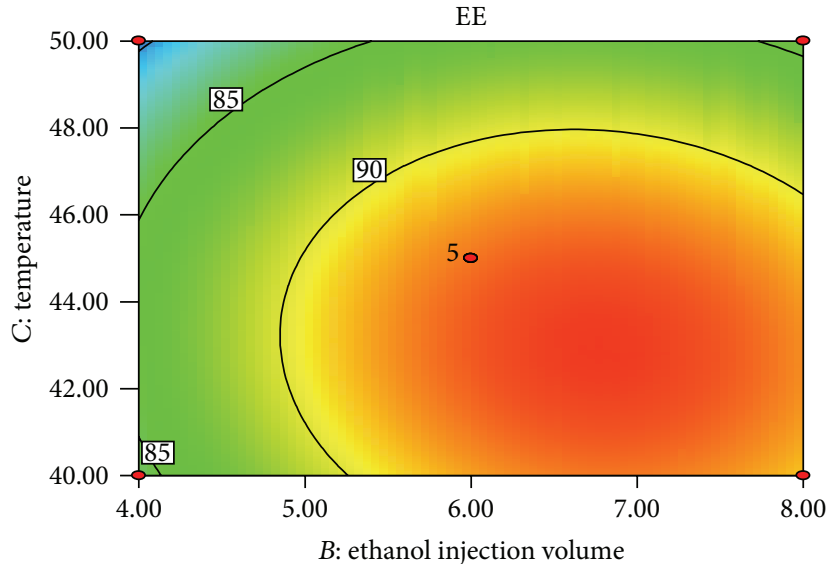

Design-Expert V8.0.6 software

Factor coding: actual

EE

- Design points

$\prod_{77}^{95}$

$X_{1}=B$ : ethanol injection volume

$X_{2}=C:$ temperature

Actual factors

A: ammonium sulfate concentration $=0.25$

$D$ : ethanol injection rate $=3.00$

(d) 


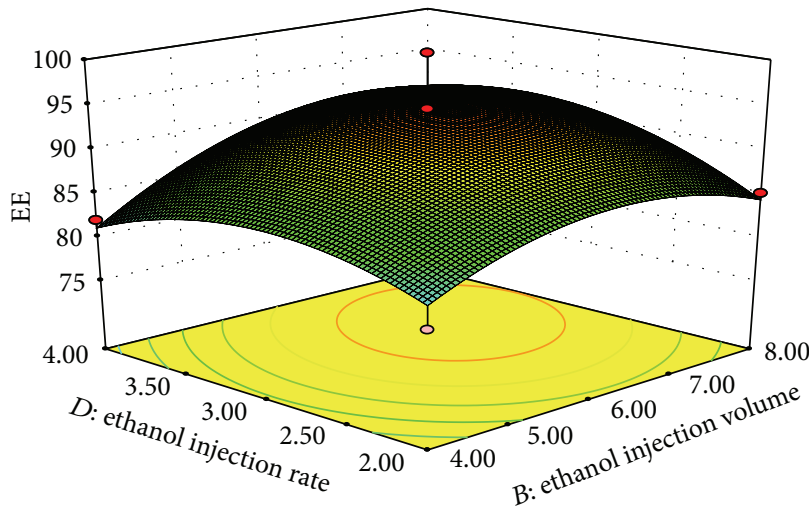

Design-Expert V8.0.6 software

Factor coding: actual

EE

- Design points above predicted value

- Design points below predicted value

$\prod_{77}^{95}$

$X_{1}=B:$ ethanol injection volume

$X_{2}=D$ : ethanol injection rate

Actual factors

A: ammonium sulfate concentration $=0.25$

$C:$ temperature $=45.00$

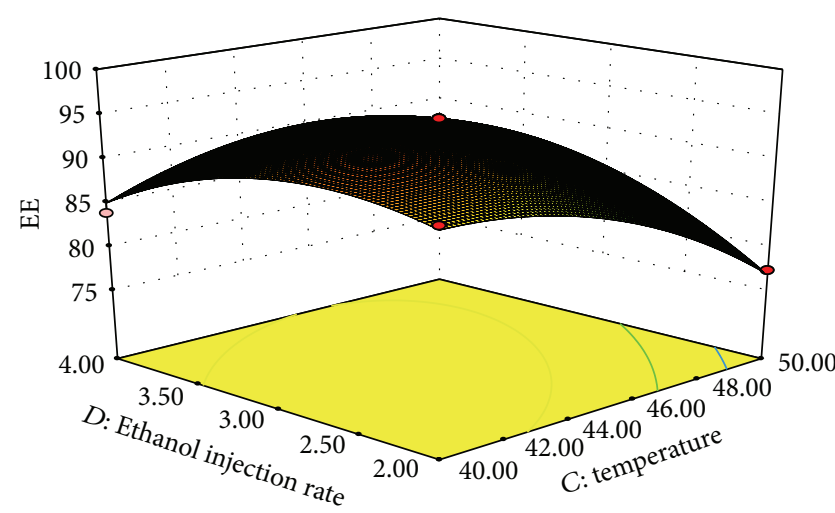

Design-Expert V8.0.6 software

Factor coding: actual

EE

- Design points above predicted value

- Design points below predicted value

! 95

$X_{1}=$ C: temperature

$X_{2}=D$ : ethanol injection rate

Actual factors

A: ammonium sulfate concentration $=0.25$

$B$ : ethanol injection volume $=6.00$

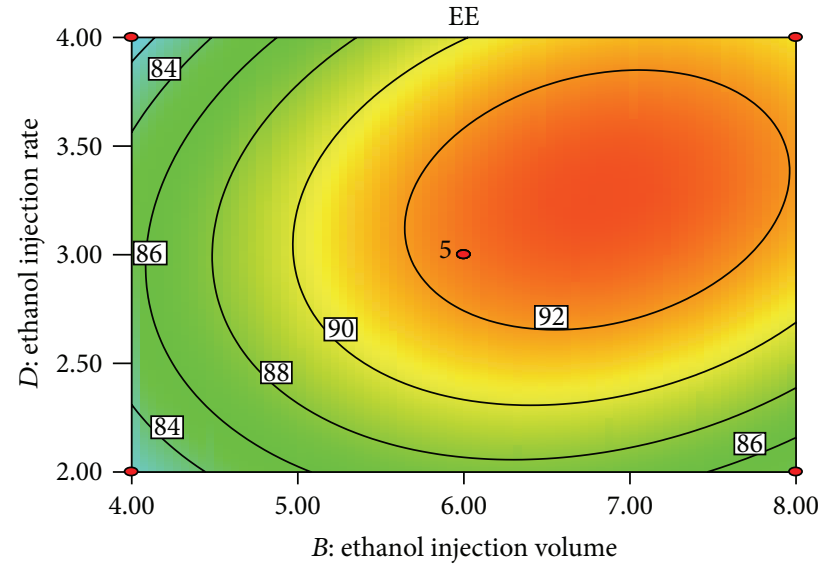

Design-Expert V8.0.6 software

Factor coding: actual

EE

- Design points

$\prod_{77}^{95}$

$X_{1}=B$ : ethanol injection volume

$X_{2}=D$ : ethanol injection rate

Actual factors

$A$ : ammonium sulfate concentration $=0.25$

$C$ : temperature $=45.00$

(e)

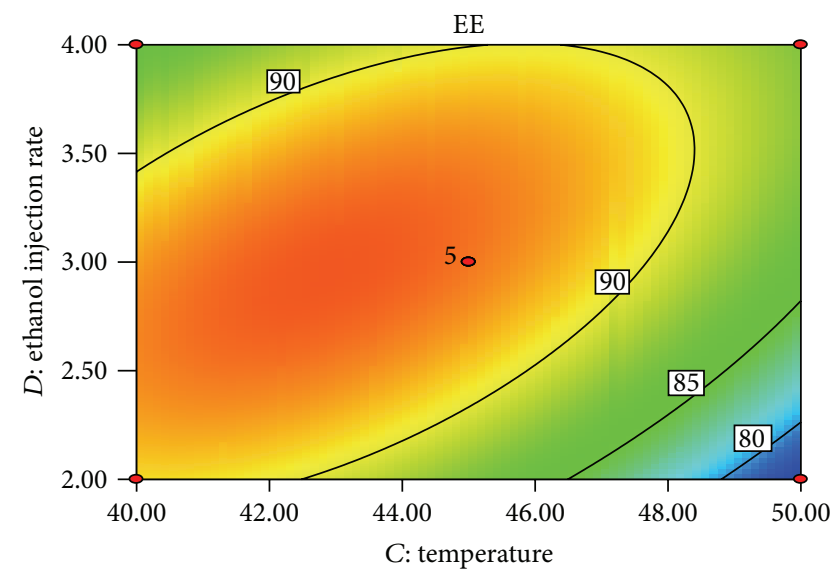

Design-Expert V8.0.6 software

Factor coding: actual

EE

- Design points

$\|_{77}^{95}$

$X_{1}=C:$ temperature

$X_{2}=D$ : ethanol injection rate

Actual factors

$A$ : ammonium sulfate concentration $=0.25$

$B$ : ethanol injection volume $=6.00$

(f)

Figure 1: Response surface (3D) and contour plots showing the interaction of different factors on the response $Y$. (a) The interaction of ammonium sulfate concentration and ethanol injection volume, (b) the interaction of ammonium sulfate concentration and temperature, (c) the interaction of ammonium sulfate concentration and ethanol injection rate, (d) the interaction of ethanol injection volume and temperature, (e) the interaction of ethanol injection volume and ethanol injection rate, and (f) the interaction of temperature and ethanol injection rate. 
TABLE 4: The results of EE of salidroside liposome PCV-2.

\begin{tabular}{|c|c|c|c|c|}
\hline Liposome & The volume ratio & $\mathrm{EE}$ & $\mathrm{TCID}_{50}$ in liposome $/ 0.2 \mathrm{~mL}$ & Total TCID $_{50} / 0.2 \mathrm{~mL}$ \\
\hline \multirow{5}{*}{ Salidroside liposome I PCV-2 } & $2: 1$ & $10 \%$ & $2.1 \times 10^{5}$ & $2.1 \times 10^{6}$ \\
\hline & $3: 1$ & $30 \%$ & $4.7 \times 10^{5}$ & $1.6 \times 10^{6}$ \\
\hline & $4: 1$ & $40 \%$ & $5.1 \times 10^{5}$ & $1.3 \times 10^{6}$ \\
\hline & $5: 1$ & $60 \%$ & $6.3 \times 10^{5}$ & $1.1 \times 10^{6}$ \\
\hline & $6: 1$ & $55 \%$ & $5.0 \times 10^{5}$ & $9.0 \times 10^{5}$ \\
\hline \multirow{5}{*}{ Salidroside liposome II PCV-2 } & $2: 1$ & $8 \%$ & $3.3 \times 10^{5}$ & $4.2 \times 10^{6}$ \\
\hline & $3: 1$ & $22 \%$ & $6.9 \times 10^{5}$ & $3.2 \times 10^{6}$ \\
\hline & $4: 1$ & $44 \%$ & $7.0 \times 10^{5}$ & $1.6 \times 10^{6}$ \\
\hline & $5: 1$ & $80 \%$ & $8.4 \times 10^{5}$ & $1.1 \times 10^{6}$ \\
\hline & $6: 1$ & $76 \%$ & $6.9 \times 10^{5}$ & $9.0 \times 10^{5}$ \\
\hline \multirow{5}{*}{ Salidroside liposome III PCV-2 } & $2: 1$ & $10 \%$ & $4.2 \times 10^{5}$ & $4.2 \times 10^{6}$ \\
\hline & $3: 1$ & $30 \%$ & $9.5 \times 10^{5}$ & $3.2 \times 10^{6}$ \\
\hline & $4: 1$ & $50 \%$ & $7.9 \times 10^{5}$ & $1.6 \times 10^{6}$ \\
\hline & $5: 1$ & $75 \%$ & $7.9 \times 10^{5}$ & $1.1 \times 10^{6}$ \\
\hline & $6: 1$ & $70 \%$ & $6.3 \times 10^{5}$ & $9.0 \times 10^{5}$ \\
\hline
\end{tabular}

TABLE 5: The characteristics of three kinds of liposomes.

\begin{tabular}{lcccc}
\hline Liposome & $\begin{array}{c}\text { Phospholipids } \\
(\mathrm{mg} / \mathrm{mL})\end{array}$ & $\begin{array}{c}\text { Salidroside } \\
(\mathrm{mg} / \mathrm{mL})\end{array}$ & $\begin{array}{c}\text { PCV-2 } \\
\left(\mathrm{TCID}_{50} / 0.2 \mathrm{~mL}\right)\end{array}$ & EE \\
\hline Salidroside liposome I PCV-2 & 30 & 0.5 & $1.1 \times 10^{6}$ & $60 \%$ \\
Salidroside liposome II PCV-2 & 60 & 0.5 & $1.1 \times 10^{6}$ & $138 \pm 22$ \\
Salidroside liposome III PCV-2 & 90 & 0.5 & $1.1 \times 10^{6}$ & $80 \%$ \\
\hline
\end{tabular}

IgG levels were shown in Figure 4. The results showed that, on the 1st and 3rd weeks after the second vaccination, the PCV2-specific IgG levels of salidroside liposome I adjuvant and salidroside liposome II adjuvant groups were significantly higher than those of the other groups $(P<0.05)$. On the 5 th, 7 th, and 9 th weeks after the second vaccination, the PCV2-specific IgG level of salidroside liposome I adjuvant group was significantly higher than that of the other groups $(P<$ 0.05 ) and the PCV-2-specific IgG level of ISA 201 adjuvant group was significantly higher than those of salidroside liposome II adjuvant and salidroside liposome III adjuvant and nonadjuvant PCV-2 groups $(P<0.05)$. On the 11th and 13th weeks after the second vaccination, the PCV-2specific IgG levels of salidroside liposome I adjuvant and ISA 201 adjuvant groups were significantly higher than those of the other groups $(P<0.05)$. The results indicated that the salidroside liposome I adjuvant and ISA 201 adjuvant could enhance the production of the PCV-2-specific IgG and the effect of salidroside liposome I adjuvant was superior to the ISA 201 adjuvant.

$\mathrm{BALB} / \mathrm{c}$ mouse was inoculated twice on days 0 and 14 with PCV-2 encapsulated in three kinds of salidroside liposome adjuvant PCV-2 vaccine, ISA 201 adjuvant PCV-2 vaccine, and PCV-2 vaccine. The levels of PCV-2-specific IgG in serum were measured on weeks $1,3,5,7,9,11$, and 13 after the second vaccination. Values were mean \pm S.E. $n=6$ mice/group. The superscripts without the same letters near the curves differ significantly $(P<0.05)$ from each other in the same week.

\section{Discussion}

Response surface methodology (RSM) is a combination of statistical and mathematical methods which is helpful for designing experiments. It is convenient for researchers to figure out complex quantitative relationships between parameters and their responses and identify the best combination of responsefactors $[20,21]$. The greatest benefit of response surface methodology is that it is able to reduce a number of experiments which are required to evaluate multiple parameters and their interactions [22]. Box-Behnken design (BBD) is one of the most common designs of the response surface methodology because of its rational design and excellent interpretation of experiments [23]. As a consequence, it has been widely used in the optimization of chemical and physical processes.

The $3 \mathrm{D}$ response surface plot and contour plot were used to explain the interaction of the variables and to identify the optimum level of each variable to reach a maximum EE yield. The response surfaces plots were presented in Figure 1. Each figure demonstrated the effect of two factors, while the other two figures were fixed at zero level.

It is judged by the shape of the contour plots whether the interactions between the corresponding variables are significant or not. According to the interaction results displayed in Figure 1, these four parameters, ammonium sulfate concentration, ethanol injection volume, temperature, and ethanol injection rate, significantly affect the EE of salidroside. 


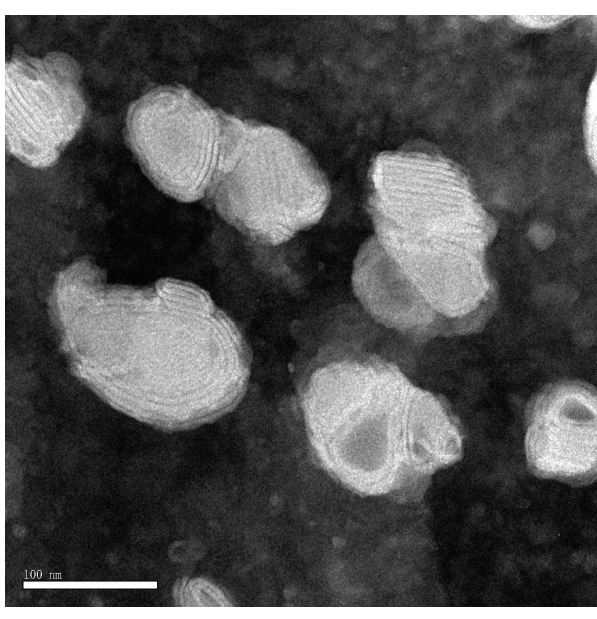

$\times 12 \mathrm{~K}$

(a)

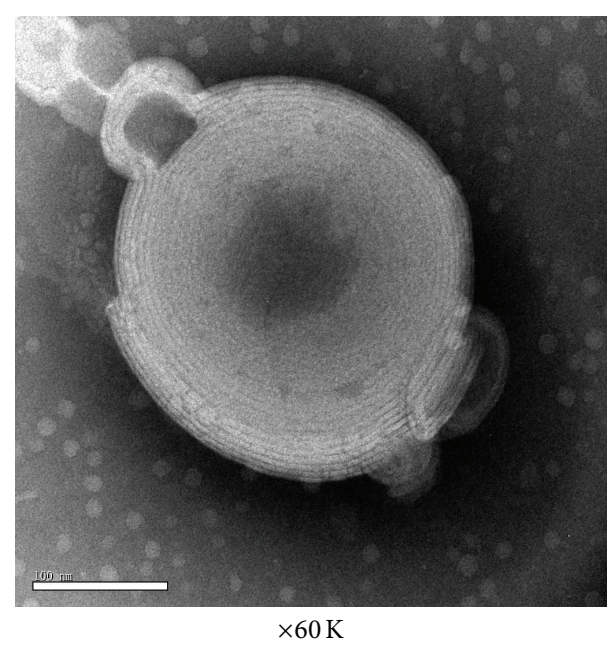

(b)

Figure 2: Transmission electron micrograph of salidroside liposome PCV-2.

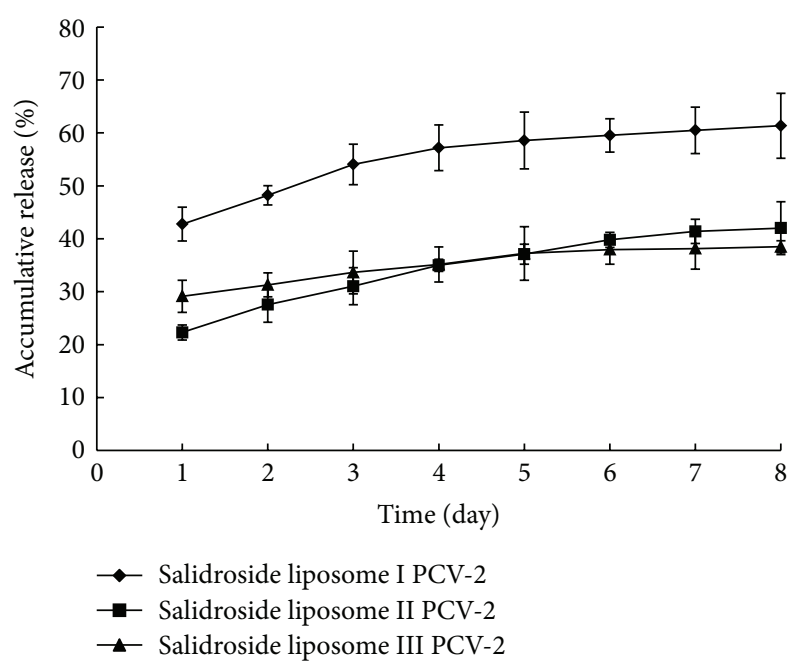

FIGURE 3: In vitro release profile of salidroside liposome PCV-2.

The optimal preparation conditions for the salidroside liposome obtained from the RSM model were as follows: ammonium sulfate concentration $(\mathrm{mol} / \mathrm{L})$ was $0.26 \mathrm{~mol} / \mathrm{L}$, ethanol injection volume $(\mathrm{mL})$ was $6.5 \mathrm{~mL}$, temperature $\left({ }^{\circ} \mathrm{C}\right)$ was $43^{\circ} \mathrm{C}$, and ethanol injection rate $(\mathrm{mL} / \mathrm{min})$ was $3 \mathrm{~mL} / \mathrm{min}$.

Besides, verification experiment was performed based on the selected optimal conditions and the actual value of $\mathrm{EE}$ from the verification experiment was very close to the predicted value. This means that the response surface method is reliable and that the data obtained from the RSM model are trusted.

Porcine circovirus type 2 (PCV-2) is the main virus involved in postweaning multisystemic wasting syndrome (PMWS) and porcine dermatitis and nephropathy syndrome (PDNS) [24-26]. The majority of pig-producing countries suffered significant economic losses because the PCV disease was the increased mortality in severely affected farms and

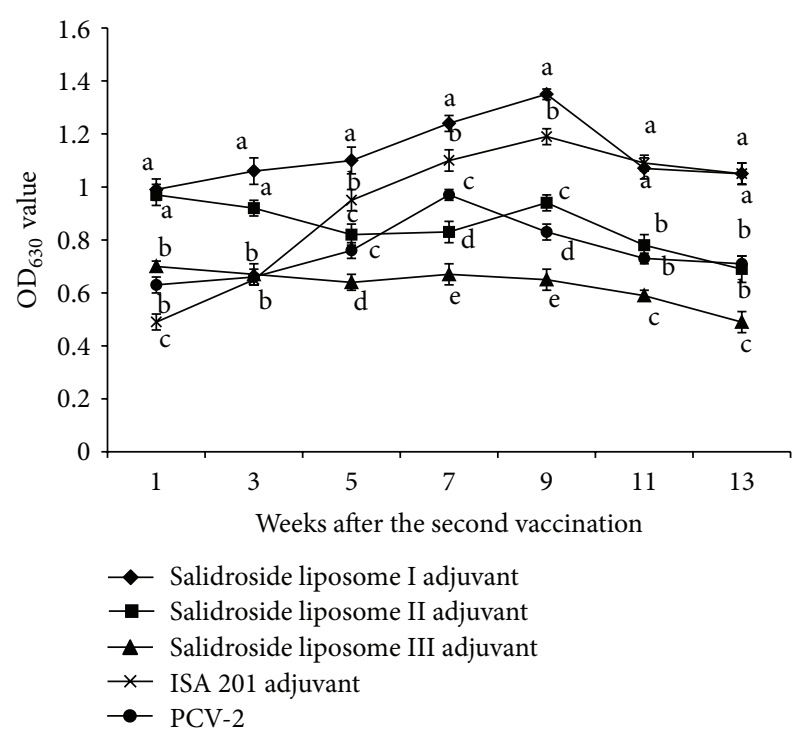

Figure 4: The PCV-2-specific IgG level of different groups after vaccination.

growth retardations in the case of subclinical infection. Therefore, it is of great importance to prevent the PCV-2 infection.

PCV-2 vaccines can provide good immunity effects only if the appropriate adjuvant was added. Nowadays, the main adjuvants used in the PCV-2 vaccine were oil emulsion adjuvants. But these adjuvants are not too much useful for vaccine because of their toxic effects, such as carcinogenic, granuloma, and cyst formation at the site of injection. Therefore, many researches were done to find alternative of the oil emulsion adjuvants and this study investigated the adjuvant activity of salidroside liposome as PCV-2 adjuvant.

The results showed that salidroside liposome as adjuvant could promote the production of PCV-2-specific IgG and 
maintain higher PCV-2-specific IgG titers for a long time. At early period of immunization (from 1 to 3 weeks), the effect of salidroside liposome I, salidroside liposome II, and salidroside liposome III adjuvants was significantly superior to ISA 201 and nonadjuvant, which indicated that the PCV2 encapsulated with salidroside liposome could more effectively induce the production of PCV-2-specific IgG. Moreover, significant difference was found between salidroside liposome I and salidroside liposome II adjuvant and ISA 201 adjuvant. From week 5 to week 9, the PCV-2-specific IgG titers of the salidroside liposome I adjuvant were significantly higher than those of ISA 201 adjuvant and those of the other groups. It indicated that humoral immunity response could be boosted when salidroside liposome I served as adjuvant. At later period of immunization (from 11 weeks to 13 weeks), the effect of salidroside liposome I adjuvant was similar to ISA 201 adjuvant, which indicated that salidroside liposome I adjuvant and ISA 201 adjuvant had controlled release. All of these results indicated that salidroside liposome I adjuvant as adjuvant could sustain for a longer effects in the whole vaccination period, which is very important to prevent the PCV-2 infection.

In conclusion, RSM was a kind of valid model to optimize the preparation conditions of salidroside liposome. The optimal preparation conditions for the salidroside liposome were as follows: ammonium sulfate concentration (mol/L) was $0.26 \mathrm{~mol} / \mathrm{L}$, ethanol injection volume $(\mathrm{mL})$ was $6.5 \mathrm{~mL}$, temperature $\left({ }^{\circ} \mathrm{C}\right)$ was $43^{\circ} \mathrm{C}$, and ethanol injection rate $(\mathrm{mL} / \mathrm{min})$ was $3 \mathrm{~mL} / \mathrm{min}$. Under these conditions, the experimental EE of salidroside liposome was $94.527 \pm 0.68 \%$. Salidroside liposome as adjuvant could rapidly induce the production of PCV-2-specific IgG and sustain higher PCV-2-specific IgG titers for a long time, and the effect of salidroside liposome was superior to ISA 201 adjuvant. These indicated that salidroside liposome could enhance the humoral immunity and had the potential to act as an effective PCV- 2 vaccine adjuvant.

\section{Abbreviations}

EE:

RSM:

PCV-2:

PBS:

BBD:

ELISA:

PMWS: $\quad \begin{aligned} & \text { Postweaning } \\ & \text { syndrome }\end{aligned}$

Encapsulation efficiency

Response surface methodology

Porcine circovirus type 2 virus

Phosphate buffered saline

Box-Behnken design

PDNS: $\quad$ Porcine dermatitis and nephropathy syndrome

BALB/c mice: An albino, laboratory-bred strain of the house mouse

MWCO: Molecular weight cutoff

BCA:

IgG: ANOVA: Bicinchoninic acid

S.E.: Immunoglobulin G

LSD:
Analysis of variance

Standard error

Least significant difference.

\section{Conflict of Interests}

The authors declare that there is no conflict of interests regarding the publication of this paper.

\section{Authors' Contribution}

Yibo Feng and Xiaojuan Zhao contributed equally to this work.

\section{Acknowledgments}

The project was supported by the National Natural Science Foundation of China (Grant no. 31372472), Special Fund for Agroscientific Research in the Public Interest (Grant no. 201303046, 201403051), Independent Innovation of Agricultural Sciences Program of Jiangsu Province (CX(13)3067), and Project Funded by the Priority Academic Program Development of Jiangsu Higher Education Institutions (PAPD). The authors are grateful to all other staff at the Institute of Traditional Chinese Veterinary Medicine of Nanjing Agricultural University for their assistance in the experiments.

\section{References}

[1] C. Cifani, B. M. V. Micioni Di, G. Vitale, V. Ruggieri, R. Ciccocioppo, and M. Massi, "Effect of salidroside, active principle of Rhodiola rosea extract, on binge eating," Physiology \& Behavior, vol. 101, no. 5, pp. 555-562, 2010.

[2] X. Zhao, Y. Lu, Y. Tao et al., "Salidroside liposome formulation enhances the activity of dendritic cells and immune responses," International Immunopharmacology, vol. 17, no. 4, pp. 1134-1140, 2013.

[3] S. E. Schriner, A. Abrahamyan, A. Avanessian et al., "Decreased mitochondrial superoxide levels and enhanced protection against paraquat in Drosophila melanogaster supplemented with Rhodiola rosea," Free Radical Research, vol. 43, no. 9, pp. 836843, 2009.

[4] A. Panossian, G. Wikman, and J. Sarris, "Rosenroot (Rhodiola rosea): traditional use, chemical composition, pharmacology and clinical efficacy," Phytomedicine, vol. 17, no. 7, pp. 481-493, 2010.

[5] H. Iinuma, K. Maruyama, K. Okinaga et al., "Intracellular targeting therapy of cisplatin-encapsulated transferrinpolyethylene glycol liposome on peritoneal dissemination of gastric cancer," International Journal of Cancer, vol. 99, no. 1, pp. 130-137, 2002.

[6] A. Schnyder and J. Huwyler, "Drug transport to brain with targeted liposomes," NeuroRx, vol. 2, no. 1, pp. 99-107, 2005.

[7] Z. ZiYu, J. LiJun, G. JianGang, L. GuangZhou, and K. Bai, "Preparation of long-circulating paclitaxel-containing liposomes and its pharmacokinetics," Food and Drug, vol. 11, no. 1, pp. 14-17, 2009.

[8] A. Sharma, E. Mayhew, L. Bolcsak et al., "Activity of paclitaxel liposome formulations against human ovarian tumor xenografts," International Journal of Cancer, vol. 71, no. 1, pp. 103-107, 1997.

[9] E. Merisko-Liversidge, S. L. McGurk, and G. G. Liversidge, "Insulin nanoparticles: a novel formulation approach for poorly water soluble Zn-insulin," Pharmaceutical Research, vol. 21, no. 9, pp. 1545-1553, 2004. 
[10] A. C. Allison and G. Gregoriadis, "Liposomes as immunological adjuvants," Nature, vol. 252, no. 5480, p. 252, 1974.

[11] M. Henriksen-Lacey, A. Devitt, and Y. Perrie, "The vesicle size of DDA: TDB liposomal adjuvants plays a role in the cell-mediated immune response but has no significant effect on antibody production," Journal of Controlled Release, vol. 154, no. 2, pp. 131-137, 2011.

[12] X. Xu, A. P. Costa, M. A. Khan, and D. J. Burgess, "Application of quality by design to formulation and processing of protein liposomes," International Journal of Pharmaceutics, vol. 434, no. 1-2, pp. 349-359, 2012.

[13] X. Xu, M. A. Khan, and D. J. Burgess, "A two-stage reverse dialysis in vitro dissolution testing method for passive targeted liposomes," International Journal of Pharmaceutics, vol. 426, no. 1-2, pp. 211-218, 2012.

[14] L. Sun and J. Du, "Revisiting the time for removing the unloaded drug by dialysis method based on a biocompatible and biodegradable polymer vesicle," Polymer, vol. 53, no. 10, pp. 2068-2073, 2012.

[15] M. Fan and S. Xu, "Adsorption and desorption properties of macroreticular resins for salidroside from Rhodiola sachalinensis A. Bor," Separation and Purification Technology, vol. 61, no. 2, pp. 211-216, 2008.

[16] C. Chen, D. Han, Y. Zhang, Y. Yuan, and X. Tang, “The freezethawed and freeze-dried stability of cytarabine-encapsulated multivesicular liposomes," International Journal of Pharmaceutics, vol. 387, no. 1-2, pp. 147-153, 2010.

[17] R. Laridi, E. E. Kheadr, R.-O. Benech, J. C. Vuillemard, C. Lacroix, and I. Fliss, "Liposome encapsulated nisin Z: optimization, stability and release during milk fermentation," International Dairy Journal, vol. 13, no. 4, pp. 325-336, 2003.

[18] R. Bo, X. Ma, Y. Feng et al., "Optimization on conditions of Lycium barbarum polysaccharides liposome by RSM and its effects on the peritoneal macrophages function," Carbohydrate Polymers, vol. 117, pp. 215-222, 2015.

[19] Y. Huang, C. Wu, Z. Liu et al., "Optimization on preparation conditions of Rehmannia glutinosa polysaccharide liposome and its immunological activity," Carbohydrate Polymers, vol. 104, no. 1, pp. 118-126, 2014.

[20] W. Cai, X. Gu, and J. Tang, "Extraction, purification, and characterization of the polysaccharides from Opuntia milpa alta," Carbohydrate Polymers, vol. 71, no. 3, pp. 403-410, 2008.

[21] W. C. Lee, S. Yusof, N. S. A. Hamid, and B. S. Baharin, "Optimizing conditions for hot water extraction of banana juice using response surface methodology (RSM)," Journal of Food Engineering, vol. 75, no. 4, pp. 473-479, 2006.

[22] A. K. Domingos, E. B. Saad, H. M. Wilhelm, and L. P. Ramos, "Optimization of the ethanolysis of Raphanus sativus (L. Var.) crude oil applying the response surface methodology," Bioresource Technology, vol. 99, no. 6, pp. 1837-1845, 2008.

[23] C.-H. Dong, X.-Q. Xie, X.-L. Wang, Y. Zhan, and Y.-J. Yao, "Application of Box-Behnken design in optimisation for polysaccharides extraction from cultured mycelium of Cordyceps sinensis," Food and Bioproducts Processing, vol. 87, no. 2, pp. 139-144, 2009.

[24] X. Ge, F. Wang, X. Guo, and H. Yang, "Porcine circovirus type 2 and its associated diseases in China," Virus Research, vol. 164, no. 1-2, pp. 100-106, 2012.

[25] A. R. Resendes, N. Majó, T. S. G. A. M. van den Ingh et al., "Apoptosis in postweaning multisystemic wasting syndrome (PMWS) hepatitis in pigs naturally infected with porcine circovirus type 2 (PCV2)," The Veterinary Journal, vol. 189, no. 1, pp. 72-76, 2011.

[26] F. M. F. Silva, A. Silva Júnior, P. M. P. Vidigal et al., "Porcine circovirus-2 viral load versus lesions in pigs: perspectives for post-weaning multisystemic wasting syndrome," Journal of Comparative Pathology, vol. 144, no. 4, pp. 296-302, 2011. 


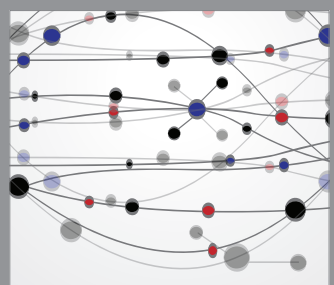

The Scientific World Journal
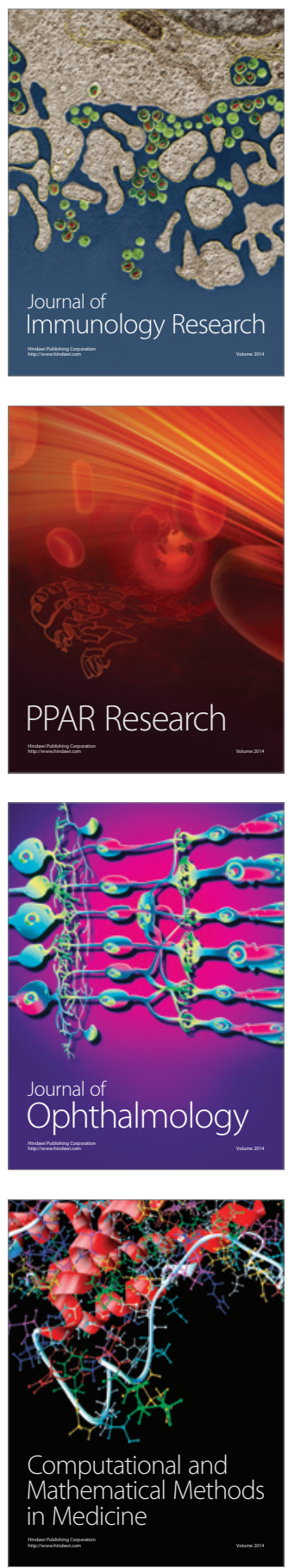

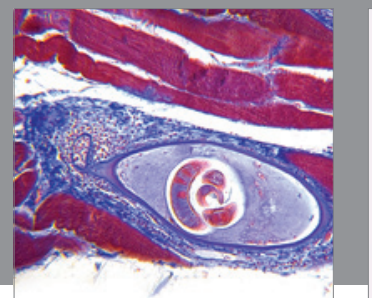

Gastroenterology

Research and Practice
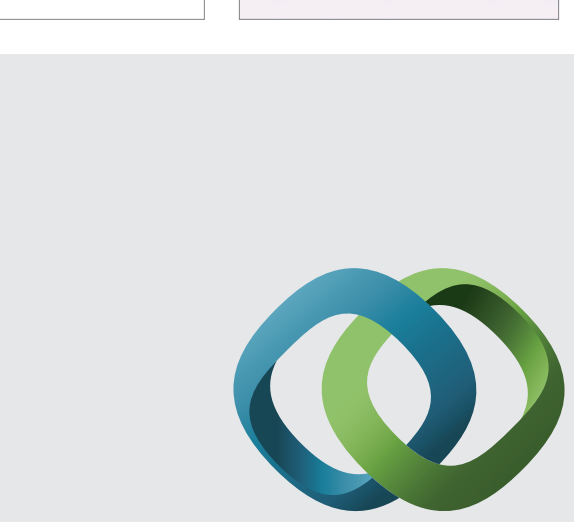

\section{Hindawi}

Submit your manuscripts at

http://www.hindawi.com
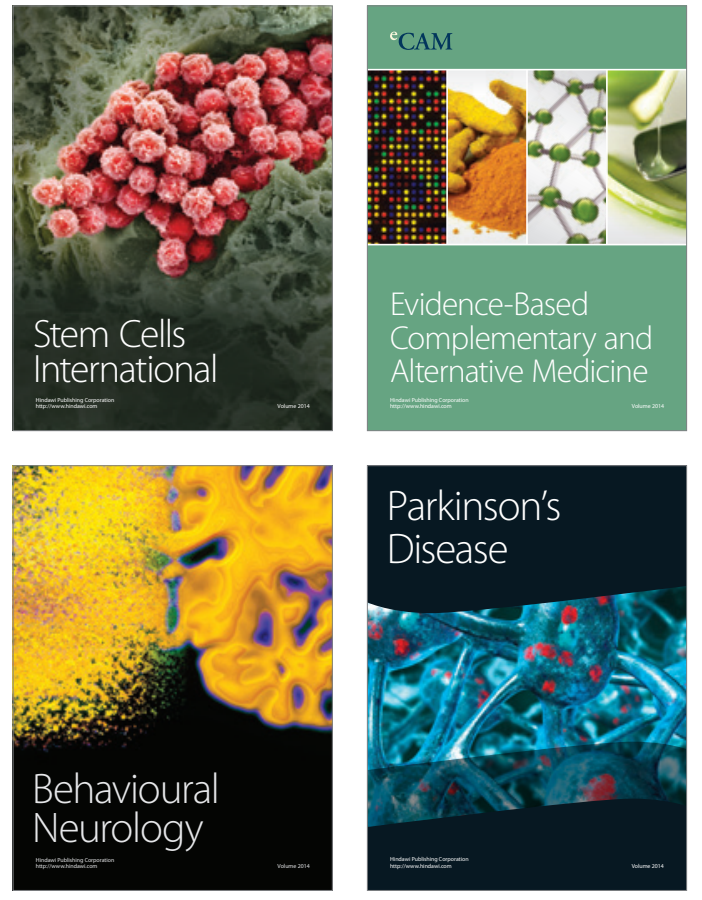
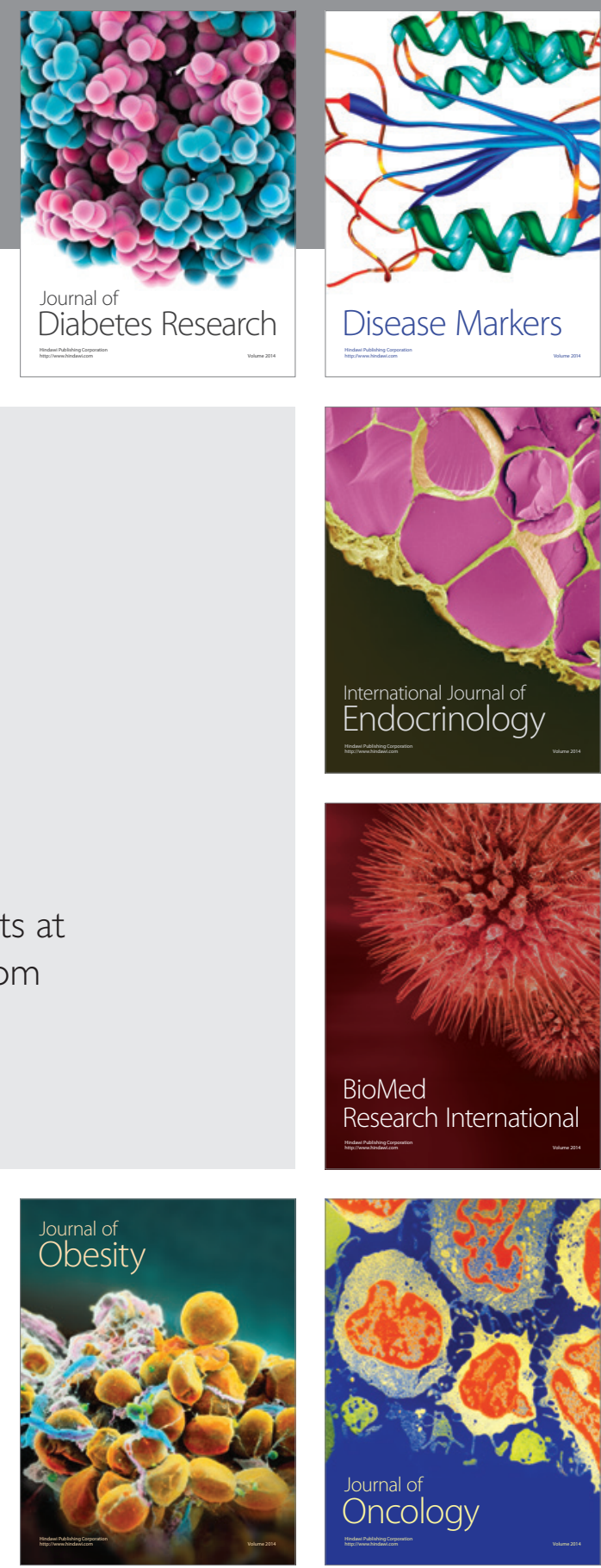

Disease Markers
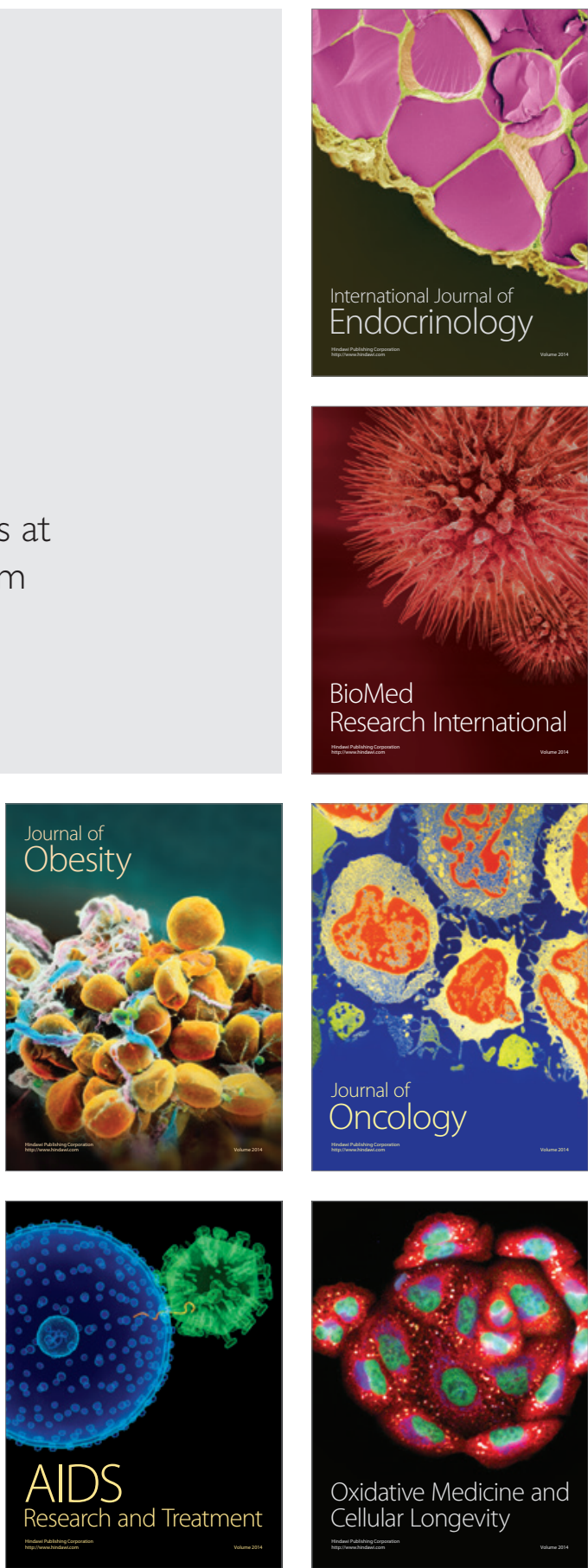